\title{
General Formula of Linear Mixed Integral Equation with Weak Singular Kernel
}

\author{
Ragab O. Abd El-Rahman \\ Department of Mathematics, Faculty of Science, Damanhur University, Egypt
}

\begin{abstract}
In this paper, we consider a mixed integral equation (MIE) of the second kind. Under certain conditions, the existence of a unique solution of is discussed and proved. The kernel of position takes asingular form, while the kernel of time is continuous. Using a quadratic numerical method, the MIE leads us to a linear system of Fredholm integral equations (SFIEs). Then,SFIEsafter using Toeplitz matrix method (TMM), tends to a linear algebraic system $(\boldsymbol{L A S})$. The existence of a unique solution of $\boldsymbol{L A S}$ is proved. Finally, numerical examples are considered, and the error, in each case, is calculated.
\end{abstract}

Keywords and phrases: Mixed integral equation, Linear system of Fredholm integral equations, Linear algebraic system, Carleman function, logarithmickernel, Toeplitz matrix method.

MSC (2010): 45B05,45E10,65R10.

\section{Introduction}

The types of integralequations (IEs)arise in a variety of applications in many fields including continuum mechanics, potential theory, geophysics, electricity and magnetism, antenna synthesis problem, mathematical physics and contact problem in the theory of elasticity, see [1-4]

In recent years, the theory of IEs has close contact with many different areas of mathematics. The following books contain many different methods to obtain the solution of the integral equation numerically, see [5-8]. The singular IEs appear in a variety of applications concerning the problems in the potential theory, see [9],wave scattering in quantum mechanics[10], diffraction problems of aero/hydroacoustis [11]. The common approach to the solution of this type involves its reduction to an equation with Cauchy and Carleman kernel.

Consider a generalized formula of linear integral equation

$$
\begin{aligned}
\mu \phi(x, t) & =\lambda \int_{\Omega} k(|x-y|) \phi(y, t) d y+\lambda \int_{0}^{t} \int_{\Omega} F(t, \tau) k(|x-y|) \phi(y, \tau) d y d \tau \\
& +\lambda \int_{0}^{t} G(t, \tau) \phi(x, \tau) d \tau+f(x, t),\left(x=\bar{x}\left(x_{1}, x_{2}, \ldots, x_{n}\right), y=\bar{y}\left(y_{1}, y_{2}, \ldots, y_{n}\right)\right) .
\end{aligned}
$$

The previous linear IEs is considered in the space $L_{2}(\Omega) \times C[0, T], T<1$, where $\Omega$ is the domain of integration with respect to position and $t \in[0, T]$ is the time. Here, in (1.1) the Fredholm integral term has a singular kernel $(|x-y|)$.While the Volterra integral term has the positive and continuous twokernels $(t, \tau)$, and $G(t, \tau)$ for all time $t, \tau \in[0, T], T<1$. The coefficient $\lambda$ is a constant, has manyphysical meaning,while, $\mu$ is a constant define the kind of the integral equation. The given function $f(x, t)$ is called the free term, and $\phi(x, t)$ is the unknown function.

Many special cases can be derived from the integral equation(1.1),

(1) If $F(t, \tau)=0$, we have

$$
\mu \phi(x, t)=\lambda \int_{\Omega} k(|x-y|) \phi(y, t) d y+\lambda \int_{0}^{t} G(t, \tau) \phi(x, \tau) d \tau+f(x, t),
$$

The above formula (1.2) is discussed in [12].

(2) If, $G(t, \tau)=0$, we obtain

$$
\mu \phi(x, t)=\lambda \int_{\Omega} k(|x-y|) \phi(y, t) d y+\lambda \int_{0}^{t} \int_{\Omega} F(t, \tau) k(|x-y|) \phi(y, \tau) d y d \tau+f(x, t) .
$$

The solution of the above formula (1.2) is obtained in [13].

(3) If,in (1.3), $\mu=0$, we have a MIE of the first kind, where [14] many spectral relationshipsare obtained in [14] 
In addition the work of Abdou et al.,in[15-17] is considered special cases of this work

In order to guarantee the existence of a unique solution of the linear equation (1.1), we assume the following conditions:

(i) The kernel ofposition satisfies the discontinuity condition

$$
\left[\int_{\Omega} \int_{\Omega} k^{2}(|x-y|) d y d x\right]^{1 / 2}=M, \quad(M \text { is a constant }) .
$$

(ii) The kernels of time $F(t, \tau)$ and $G(t, \tau)$ belong to the class $C[0, T], 0 \leq \tau \leq t \leq T<1$, and satisfy for the constants, $N>N_{1}, N>N_{2}$, the conditions

$$
|F(t, \tau)| \leq N_{1},|G(t, \tau)| \leq N_{2} \quad, \forall t, \tau \in[0, T] .
$$

(iii) The given function $f(t, \tau)$, with its partial derivatives with respect to $x$ and $t$, are continuous in $L_{2}(\Omega) \times C[0, T], T<1$, and its norm is defined as

$$
\|f(x, t)\|=\max _{0 \leq t \leq T} \int_{0}^{t}\left[\int_{\Omega} f^{2}(x, \tau) d x\right]^{\frac{1}{2}} d \tau=H \quad, \quad(H \text { is a constant }) .
$$

(iv) The unknown function $\phi(x, t)$ satisfies Lipschitz condition for the first argument and Holder condition for the second argument.

In this paper, the existence of a unique solution of the IE (1.1) is discussed and proved. A numerical method is used to translate the MIE (1.1) to a system of FIEs of the second kind.Then the existence of a unique solution of this system is proved. The TMMis used to obtain a LAS, where the existence of a unique solution of this system will be proved. Finally, we obtain, numerically the solution of the LAS when the kernel takes the weakly forms $\left(k(|x-y|)=\ln |x-y|, k(|x-y|)=|x-y|^{-v} \quad, 0<v<1 \quad\right)$. Moreover, numerical results are obtained and the error estimate, in each case, is computed.

\section{Quadratic Numerical Method}

In this section,a quadratic numerical method is used to represent (1.1) as a SFIEs. For this, we divide the interval $[0, T], 0 \leq t \leq T<1$

as $0=t_{0}<t_{1}<t_{2}<\cdots<t_{l}<\cdots<t_{p}=T$, where $t=t_{l}, l=1,2, \ldots, p$; to get

$$
\begin{gathered}
\mu \phi\left(x, t_{l}\right)=\lambda \int_{\Omega} k(|x-y|) \phi\left(y, t_{l}\right) d y+\lambda \int_{0}^{t_{l}} \int_{\Omega} F\left(t_{l}, \tau\right) k(|x-y|) \phi(y, \tau) d y d \tau \\
+\lambda \int_{0}^{t_{l}} G\left(t_{l}, \tau\right) \phi(x, \tau) d \tau+f\left(x, t_{l}\right) .
\end{gathered}
$$

Using the quadrature formula,see Atkinson [18] we have

$$
\mu_{l} \phi_{l}(x)=\lambda_{l} \int_{\Omega} k(|x-y|) \phi_{l}(y) d y+H_{l}(x)+E_{p, l}(x), E_{p, l}(x)=\max _{l_{1}, l_{2}}\left\{E_{p, l_{1}}, E_{p, l_{2}}\right\},
$$

where $\mu_{l}=\mu-\lambda w_{l_{2}} G_{l, l_{2}}, \lambda_{l}=\lambda\left(1+u_{l} F_{l, l_{1}}\right)$ ，

and

$$
H_{l}(x)=\lambda \sum_{j=0}^{l_{2}-1} w_{j} G_{l, j} \phi_{j}(x)+\lambda \sum_{j=0}^{l_{1}-1} u_{j} F_{l, j} \int_{\Omega} k(|x-y|) \phi_{j}(y) d y+f_{l}(x),
$$

Here, we used the following notations:

$$
\begin{gathered}
\phi_{l}(x)=\phi\left(x, t_{l}\right), \quad F_{l, j}=F\left(t_{l}, t_{j}\right), \quad G_{l, j}=G\left(t_{l}, t_{j}\right), \quad f_{l}(x)=f\left(x, t_{l}\right), \\
(l=0,1,2, \ldots, p, \quad 0 \leq j \leq l) \quad(2.4)
\end{gathered}
$$

The characteristic points $u_{j}, w_{j}$ and the errors $E_{p, l_{1}}, E_{p, l_{2}}, p \approx l, l_{1}, l_{2}<l$, are depend on the number of the derivatives of $F(t, \tau)$ and $G(t, \tau)$, respectively , tot $\in[0, T]$.

The existence of a unique solution of the (2.2), can easily be provedunder the condition (i) and the following conditions

1) $\max _{j}\left\|f_{j}(x)\right\|_{L_{2}(\Omega)} \leq \mathcal{Q} \quad, \quad \mathcal{Q}$ is a constant , 
2) For the constants $L>\left\{Q_{1}, Q_{2}\right\}$, we have $\sum_{j=0}^{l-1} \max _{j}\left|u_{j} F_{l, j}\right| \leq Q_{1}, \sum_{j=0}^{l-1} \max _{j}\left|u_{j} G_{l, j}\right| \leq Q_{2}$,

\section{The Toeplitz Matrixmethod, See Abdou Et Al. [16, 19]}

Write theSFIEs (2.2), when $\Omega=[-a, a]$, as

$$
\mu_{l} \phi_{l}(x)=\psi_{l}(x)+\lambda_{l} \int_{\Omega} k(|x-y|) \phi_{l}(y) d y .
$$

Write the integral term in the form

$$
\int_{-a}^{a} k(|x-y|) \phi_{l}(y) d y=\sum_{n=-N}^{N+1} \int_{n h}^{n h+h} k(|x-y|) \phi_{l}(y) d y \quad,\left(h=\frac{a}{N}\right) .
$$

Then, approximate the integral in theright hand side of (3.2) by

$$
\int_{n h}^{n h+h} k(|x-y|) \phi_{l}(y) d y=A_{n}^{(l)}(x) \phi_{l}(n h)+B_{n}^{(l)}(x) \phi_{l}(n h+h)+E_{N, n}^{(l)},
$$

where $E_{N, n}^{(l)}$ is the estimate error . Using the principal idea of the TMMby assuming in (3.3), $\phi_{l}(x)=1, x$, respectively, in this case $E_{N, n}=0$. Hence, we have two formulas of two unknown functions $A_{n}^{(l)}(x)$ and $B_{n}^{(l)}(x)$ . By solving the results, the functions $A_{n}^{l}(x)$ and $B_{n}^{l}(x)$ take the forms

where the values of $I(x)$ and $J(x)$ are

$$
A_{n}^{(l)}(x)=\frac{[(n h+h) I(x)-J(x)]}{h}, B_{n}^{(l)}(x)=\frac{[J(x)-n h I(x)]}{h},
$$

$$
I(x)=\int_{n h}^{n h+h} k(|x-y|) d y \quad, \quad J(x)=\int_{n h}^{n h+h} y k(|x-y|) d y,
$$

Therefore, the relation (3.2), becomes

$$
\int_{-a}^{a} k(|x-y|) \phi_{l}(y) d y=\sum_{n=-N}^{N} G_{n}^{(l)}(x) \phi_{l}(n h),
$$

where

$$
G_{n}^{(l)}(x)=\left\{\begin{array}{cc}
A_{-N}^{(l)} & , n=-N \\
A_{n}^{(l)}(x)+B_{n-1}^{(l)}(x) & ,-N<n<N \\
B_{N-1}^{(l)}(x) & , n=N \leq l \leq p
\end{array}\right.
$$

The integral equation (3.1), after putting $=m h$, becomes

$$
\mu_{l} \phi_{l, m}-\lambda_{l} \sum_{n=-N}^{N} Y_{n, m}^{(l)} \phi_{l, n}=\psi_{l, m} \quad, \quad l=0,1,2, \ldots, p \quad,
$$

The solution of the formula (3.8) takes the form

$$
\phi_{l, m}=\left[\mu_{l} I-\lambda_{l} Y_{n, m}^{(l)}\right]^{-1} \psi_{l, m} \quad, \quad\left|\mu_{l} I-\lambda_{l} Y_{n, m}^{(l)}\right| \neq 0 .
$$

The formula (3.8) or (3.9) represents a SAEs, where $\phi_{m}$ is a vector of $2 N+1$ elements, $I$ is the unit matrix of order $n \times m$ and $\mathrm{Y}_{n, m}$ is a matrix whose elements are given by

$\begin{array}{cc}Y_{n, m}^{(l)}=G_{n-m}+P_{n, m} \\ G_{n-m}=A_{n}^{(l)}(m h)+B_{n-1}^{(l)}(m h), & -N \leq n \leq N \quad(3.10)\end{array}$

$$
\begin{aligned}
& Y_{n, m}^{(l)}=G_{n-m}+P_{n, m} \\
& -N \leq n \leq N \quad(3.10)
\end{aligned}
$$

The matrix $G_{n-m}$ is a Toeplitz matrix of order $2 N+1$, where $-N \leq m, n \leq N$ and the elements of the second matrix are zeros except the elements of the first and last rows (columns). We can evaluate the values of the first row by substituting in $B_{n-1}^{(l)}(m h) \quad n=-N$ and $=-N+i, 0 \leq i \leq 2 N$, and the values of the last row (column) by substituting, in $A_{n}^{(l)}(m h) \quad n=-N$ and $m=-N+i$.

Definition1: The TMM is said to be convergent of order $r$ in $[-a, a]$, if for $N$ sufficiently large, there exist a constant $D>0$ independent of $N$ such that

$$
\left\|\phi(x)-\phi_{N}(x)\right\| \leq D N^{-r},
$$

The error term $E_{N, n}^{(l)}$ is determined from the following formula 
$E_{N, n}^{(l)}=\left|\int_{\mathrm{nh}}^{n h+h} y^{2} k(|x-y|) d y-A_{n}^{(l)}(x)(n h)^{2}-B_{n}^{(l)}(x)(n h+h)^{2}\right|=O\left(h_{l}^{3}\right),\left(h_{l}^{4} \rightarrow 0\right)$.

The existence of a unique solution of the algebraic systemin $\ell^{\infty}$ :

For thisaim, we write the system (3.8) in the operator form

$$
\begin{gathered}
\bar{T} \phi_{l, m}=T \phi_{l, n}+\psi_{l, m} \quad, T \phi_{l, n}=\frac{\lambda_{l}}{\mu_{l}} \sum_{n=-N}^{N} Y_{n, m}^{(l)} \phi_{l, n}, \quad \mu_{l} \neq 0 \\
\psi_{l, m}=\frac{\lambda_{l}}{\mu_{l}} \sum_{j=0}^{l-1} u_{j}\left[F_{i, j} \sum_{m=-N}^{N} Y_{j, m}^{(l)} \phi_{j, n}+G_{i, j} \phi_{j, m}\right]+\frac{1}{\mu_{l}} f_{l, m},
\end{gathered}
$$

Then, the following lemma can be proved.

Lemma 1:If the kernel of position satisfies the conditions:

$$
\begin{array}{r}
(i): k(|x-y|) \in L_{q}, \quad(q>1),(i i): \lim _{x^{\prime} \rightarrow x}\left\|k\left(\left|x^{\prime}-y\right|\right)-k(|x-y|)\right\|_{L_{q}}=0 \\
x, x^{\prime} \in[-a, a],
\end{array}
$$

we have

a) $\max _{0 \leq l \leq p} \operatorname{Sup}_{N} \sum_{n=-N}^{N}\left|Y_{n, m}^{(l)}(x)\right|<c, c$ is a constant ,

$$
\text { b) } \lim _{m \rightarrow m} \max _{0 \leq l \leq p} \operatorname{Sup}_{N} \sum_{n=-N}^{N}\left|Y_{n, m}^{(l)},-Y_{n, m}^{(l)}\right|=0, \quad \forall 0 \leq l \leq p .
$$

Proof: Firstly, we go to prove (a) of Eq. (3.15). S0, from the first formula of (3.4), we obtain

$$
\left|A_{n}^{(l)}(x)\right|=\left[(n h+h) \int_{n h}^{n h+h}|k(|x-y|)| d y-\int_{n h}^{n h+h}|y||k(|x-y|)| d y\right] /|h| .
$$

Then, using the first condition (i) of Eq. (3.14), we deduce that there exist a small constant

$$
z_{1} \text {, such thatmax } \sum_{0 \leq l \leq p} \sum_{n=-N}^{N}\left|A_{n}^{(l)}(x)\right| \leq z_{1}, \forall N \text {. Since each term in this inequality is }
$$

boundedabove, hence for $x=m h$, we write

$$
\max _{0 \leq l \leq p} \operatorname{Sup}_{N} \sum_{n=-N}^{N}\left|A_{n}^{(l)}(m h)\right| \leq z_{1} .
$$

Similarly, from the second formula of (3.4), we can find a small constant $z_{2}$, such that

$$
\max _{0 \leq l \leq p} \operatorname{Sup}_{N} \sum_{n=-N}^{N}\left|B_{n}^{(l)}(m h)\right| \leq z_{2} .
$$

Therefore,from the relations (3.7), (3.16) and (3.17),there exists a small constant $z \leq\left(z_{1}+z_{2}\right)$, such that the first inequality of (3.15) is proved.

To prove (b), using the first formula of (3.4), for $x, x^{\prime} \in[-a, a]$, and applying Hölder inequality, then summing from $n=-N$ to $n=N$, to have

$$
\max _{0 \leq l \leq p} \sum_{n=-N}^{n=N}\left|A_{n}^{(l)}\left(x^{\prime}\right)-A_{n}^{(l)}(x)\right| \leq \sum_{n=-N}^{n=N} q_{n}\left\|k\left(\left|x^{\prime}-y\right|\right)-k(|x-y|)\right\|_{L_{q}} .
$$

Putting $=m h, x^{\prime}=m^{\prime} h$, then using the condition (3.14), when $x^{\prime} \rightarrow x$, we get

$$
\lim _{m^{\prime} \rightarrow m 0 \leq l \leq p} \max _{N} \operatorname{Sup}_{n=-N} \sum_{n=N}\left|A_{n}^{(l)}\left(m^{\prime} h\right)-A_{n}^{(l)}(m h)\right|=0 .
$$

Similarly, in view of the second formula of (3.4), we have

$$
\lim _{m^{\prime} \rightarrow m 0 \leq l \leq p} \operatorname{map}_{N} \sum_{n=-N}^{n=N}\left|B_{n}^{(l)}\left(m^{\prime} h\right)-B_{n}^{(l)}(m h)\right|=0 .
$$

Hence, from (3.18) and (3.19), the second inequality of (3.15) is proved.o

Now, under the two conditions of lemma 3.1 and the following condition 
(c) $\max _{0 \leq l \leq p} \operatorname{Sup}_{m} \sum_{n=-N}^{N}\left|f_{n, m}\right| \leq H \quad, \quad(H$ is a constant $)$

We can state the following

Theorem 1: The LASof (3.8) or (3.13), has a unique solution in the Banachspace $\ell^{\infty}$, under the following condition

$$
\left|\lambda_{l}\right|<\left|\mu_{l}\right| /(c+L c+L),(L \text { is defined by condition } 2) \text {. }
$$

To prove thistheorem, we consider the following twolemmas.

Lemma 2:The operator $\bar{T}$ of Eq. (3.13) is bounded.

Proof: Let $V$ be the set of all sequences $\Phi=\left\{\phi_{l, m}\right\}$ in $\ell^{\infty}$ such that $\|\Phi\|_{\ell^{\infty}} \leq \beta, \beta$ is aconstant. Define the norm of the operator $\bar{T}$ Фin Banach space $\ell^{\infty}$ by

$\|\bar{T} \Phi\|=\max _{0 \leq l \leq p} \operatorname{Sup}_{m}\left|\bar{T} \phi_{l, m}\right|, \quad$ for each integer $m$.

Hence, the integral operator (3.13) takes the form

$$
\begin{gathered}
\max _{0 \leq l \leq p} \operatorname{Sup}_{m}\left|\bar{T} \phi_{l, m}\right| \leq\left|\frac{\lambda_{l}}{\mu_{l}}\right|\left\{\max _{0 \leq l \leq p} \sum_{n=-N}^{N}\left|Y_{n, m}^{(l)}\right| \operatorname{Sup}_{n}\left|\phi_{l, n}\right|+\right. \\
\left.\max _{0 \leq l \leq p} \sum_{j=0}^{l}\left[\left|u_{j} F_{i, j}\right| \sum_{n=-N}^{N}\left|Y_{n, m}^{(l)}\right| \operatorname{Sup}_{n}\left|\phi_{l, n}\right|+\left|u_{j} G_{l, j}\right| \operatorname{Sup}_{m}\left|\phi_{l, m}\right|\right]\right\}+\left|\frac{1}{\mu_{l}}\right| \max _{0 \leq l \leq p} \operatorname{Sup}_{m}\left|f_{l, m}\right|
\end{gathered}
$$

Using condition (2), and Lemma 3.1 and condition (c), we have

$$
\begin{aligned}
& \max _{0 \leq l \leq p} \operatorname{Sup}_{m}\left|\bar{T} \phi_{m}\right| \leq\left|\frac{\lambda_{l}}{\mu_{l}}\right|\left\{\max _{0 \leq l \leq p} \operatorname{Sup}_{n} \sum_{n=-N}^{N}\left|Y_{n, m}^{(l)}\right|\|\Phi\|_{\ell^{\infty}}+\right. \\
& \left.\sum_{j=0}^{l}\left[L \max _{0 \leq l \leq p} \operatorname{Sup}_{n} \sum_{n=-N}^{N}\left|Y_{n, m}^{(l)}\right|\|\Phi\|_{\ell^{\infty}}+L\|\Phi\|_{\ell^{\infty}}\right]\right\}+\left|\frac{1}{\mu_{l}}\right| H
\end{aligned}
$$

Finally, with the aid of (3.21), we have

$$
\|\mathrm{T} \Phi\|=\max _{0 \leq l \leq p} \operatorname{Sup}_{m}\left|\bar{T} \phi_{l, m}\right| \leq \alpha_{2}\|\Phi\|_{\ell^{\infty}}+\frac{H}{\left|\mu_{l}\right|} \quad, \quad\left(\alpha_{2}=\left|\lambda_{l} / \mu_{l}\right|(c+L c+L)\right) .
$$

The inequality (3.22) shows that the operator $\bar{T}$ maps the set $V$ into itself, where $\beta=H /\left|\mu_{l}\right|\left(1-\alpha_{2}\right)$. Hence, we deduce $\alpha_{2}<1$. In addition, the inequality (3.22) involves the boundedness of the operators $T$ and $\bar{T}$.

Lemma 3: The operator $\bar{T}$ is continuous and contractive operator.

Proof:For the two sets $\Phi=\left\{\phi_{l, m}\right\}$, and, $\bar{\Phi}=\left\{\overline{\phi_{l, m}}\right\}$, we have

$$
\begin{aligned}
\max _{0 \leq l \leq p} & \operatorname{Sup}_{m}\left|\bar{T} \phi_{l, m}-\bar{T} \overline{\phi_{l, m}}\right| \leq\left|\frac{\lambda}{\mu}\right|\left\{\max _{0 \leq l \leq p} \sum_{n=-N}^{N}\left|Y_{n, m}^{(l)}\right| \operatorname{Sup}_{n}\left|\phi_{l, n}-\overline{\phi_{l, n}}\right|+\max _{0 \leq l \leq p} \sum_{j=0}^{l}\left[\left|u_{j} F_{i, j}\right|\right.\right. \\
& \left.\left.\sum_{n=-N}^{N}\left|Y_{n, m}^{(l)}\right| \operatorname{Sup}_{n}\left|\phi_{l, n}-\overline{\phi_{l, n}}\right|+\left|u_{j} G_{l, j}\right| \operatorname{Sup}_{m}\left|\phi_{l, m}-\overline{\phi_{l, m}}\right|\right]\right\} \leq\left|\frac{\lambda}{\mu}\right|\left\{\max _{0 \leq l \leq p} \operatorname{Sup}_{n}\right. \\
& \left.\sum_{n=-N}^{N}\left|Y_{n, m}^{(l)}\right|\|\Phi-\bar{\Phi}\|+\sum_{j=0}^{l}\left[L \max _{0 \leq l \leq p} \operatorname{Sup}_{n} \sum_{n=-N}^{N}\left|Y_{n, m}^{(l)}\right|\|\Phi-\bar{\Phi}\|+L\|\Phi-\bar{\Phi}\|\right]\right\} .
\end{aligned}
$$

Hence, we get

$$
\|\bar{T} \Phi-\bar{T} \bar{\Phi}\|_{\ell^{\infty}} \leq \alpha_{2}\|\Phi-\bar{\Phi}\|_{\ell^{\infty}} \quad, \quad \alpha_{2}<1 .
$$

The previous inequality tells us that $\bar{T}$ is a continuous operator and under condition (3.20) it is a contractive operator in $\ell^{\infty}$.Hence, the Theorem is proved.d

Definition 2: The estimate local error $E_{s, n}$ of (3.3) is determined as

$$
\phi_{l}(x)-\left(\phi_{l}(x)\right)_{s}=\sum_{n=-s}^{s} Y_{n, m}^{(l)}\left[\phi_{l}(n h)-\phi_{l, s}(n h)\right]+E_{s, n}^{(l)} \quad, \quad(x=m h)
$$


where $\left(\phi_{l}(x)\right)_{s}$ is the approximate solution of equation (3.1).

Corollary1:Assume the hypothesis of theorem 2are verified , then $\lim _{s \rightarrow \infty} E_{s, n}^{(l)}=0$.

Proof:In view of formula (3.23), we have

$$
\left|E_{s, n}^{(l)}\right| \leq \max _{0 \leq l \leq p} \operatorname{Sup}_{m}\left|\phi_{l}(m h)-\left(\phi_{l}(m h)\right)_{s}\right|+\left\|\Phi-\Phi_{s}\right\|_{\ell^{\infty}} \operatorname{Sup}_{n} \sum_{n=-s}^{s}\left|Y_{n, m}^{(l)}\right| .
$$

The above inequality is true for each integer , and by condition (b), we get

$$
\left\|E_{s, n}^{(l)}\right\|_{\ell^{\infty}} \leq(1+c)\left\|\Phi-\Phi_{s}\right\|_{\ell^{\infty}} \quad \text {, for each } s .
$$

Since $\left\|\Phi-\Phi_{s}\right\| \rightarrow \infty$ as $s \rightarrow \infty$ then $E_{s, n}^{(l)} \rightarrow 0$ as $s \rightarrow \infty$.

Finally, it is convenient to consider the following theorem, which proves the convergence of the sequence of approximate solution $\left\{\left(\phi_{l}(m h)\right)_{z, s}\right\}$ to the exact solution of equation (1.1) in Banachspace $L_{2}(\Omega) \times C[0, T]$.

Definition 3:The following relationdetermines the totalerror $E_{Z, S}$ :

$$
\begin{gathered}
E_{z, s}=\int_{-a}^{a} k(|x-y|) \phi(y, t) d y+\int_{0}^{t} \int_{-a}^{a} F(t, \tau) k(|x-y|) \phi(y, \tau) d y d \tau+ \\
\left.\int_{0}^{t} G(t, \tau) \phi(x, \tau) d \tau-\sum_{j=0}^{l} u_{l}\left[F_{i, j} \sum_{n=-s}^{s} Y_{n, m}^{(l)} \phi_{j, n}+G_{j, l} \phi_{j, m}\right]-\sum_{n=-s}^{s} Y_{n, m}^{(l)} \phi_{j, n}\right] . \\
\text { When } z, s \rightarrow \infty, \text { the sum } \sum_{l=0}^{i} u_{l}\left[F_{i, l} \sum_{n=-s}^{s} Y_{n, m} \phi_{l, n}+G_{i, l} \phi_{l, m}\right]+\sum_{n=-s}^{s} Y_{n, m} \phi_{l, n} \rightarrow \\
\int_{-a}^{a} k(|x-y|) \phi(y, t) d y+\int_{0}^{t} \int_{-a}^{a} F(t, \tau) k(|x-y|) \phi(y, \tau) d y d \tau+\int_{0}^{t} G(t, \tau) \phi(x, \tau) d \tau
\end{gathered}
$$

and the solution of the algebraic system becomes the solution of the equation (1.1).

Consider the mixed integral equation

\section{Application}

$$
\begin{array}{r}
\mu \phi(x, t)=\lambda \int_{\Omega} \phi(y, t) k(|x-y|) d y+\lambda \int_{0}^{t} \int_{\Omega} t^{2} \tau \phi(y, \tau) k(|x-y|) d y d \tau \\
+\lambda \int_{\Omega} t \tau^{2} \phi(x, \tau) d \tau+f(x, t) . \quad\left(\phi(y, t)=x^{2}+t^{2}\right)
\end{array}
$$

Example(1): Consider $k(|x-y|)=|x-y|^{-v} ; 0<v<1$, at $\lambda=0.5152 ; \mu=1 ; T=0.2 ; n=41$

The approximate solution and the estimate error, in each cases, for $v=0.12$ and $v=0.73$ are calculated in table (1).

Table1

\begin{tabular}{|l|l|l|l|l|l|}
\hline \multirow{2}{*}{$\boldsymbol{*}$} & \multirow{2}{*}{ Exact } & \multicolumn{2}{|c|}{$\boldsymbol{v}=\mathbf{0 . 1 2}$} & \multicolumn{2}{c|}{$\boldsymbol{v}=\mathbf{0 . 7 3}$} \\
\cline { 3 - 6 } & & App. Sol. & Error & App. Sol. & Error \\
\hline-1 & $4.00000 \mathrm{E}-04$ & $3.63253 \mathrm{E}-04$ & $3.67468 \mathrm{E}-05$ & $3.08591 \mathrm{E}-04$ & $9.14093 \mathrm{E}-05$ \\
\hline-0.8 & $2.56000 \mathrm{E}-04$ & $2.73278 \mathrm{E}-04$ & $1.72775 \mathrm{E}-05$ & $2.24550 \mathrm{E}-04$ & $3.14502 \mathrm{E}-05$ \\
\hline-0.6 & $1.44000 \mathrm{E}-04$ & $1.61339 \mathrm{E}-04$ & $1.73390 \mathrm{E}-05$ & $1.10959 \mathrm{E}-04$ & $3.30406 \mathrm{E}-05$ \\
\hline-0.4 & $6.40000 \mathrm{E}-05$ & $8.14052 \mathrm{E}-05$ & $1.74052 \mathrm{E}-05$ & $3.00103 \mathrm{E}-05$ & $3.39897 \mathrm{E}-05$ \\
\hline-0.2 & $1.60000 \mathrm{E}-05$ & $3.34485 \mathrm{E}-05$ & $1.74485 \mathrm{E}-05$ & $-1.85140 \mathrm{E}-05$ & $3.45140 \mathrm{E}-05$ \\
\hline 0 & $0.00000 \mathrm{E}+00$ & $1.74632 \mathrm{E}-05$ & $1.74632 \mathrm{E}-05$ & $-3.46828 \mathrm{E}-05$ & $3.46828 \mathrm{E}-05$ \\
\hline 0.2 & $1.60000 \mathrm{E}-05$ & $3.34485 \mathrm{E}-05$ & $1.74485 \mathrm{E}-05$ & $-1.85140 \mathrm{E}-05$ & $3.45140 \mathrm{E}-05$ \\
\hline 0.4 & $6.40000 \mathrm{E}-05$ & $8.14052 \mathrm{E}-05$ & $1.74052 \mathrm{E}-05$ & $3.00103 \mathrm{E}-05$ & $3.39897 \mathrm{E}-05$ \\
\hline 0.6 & $1.44000 \mathrm{E}-04$ & $1.61339 \mathrm{E}-04$ & $1.73390 \mathrm{E}-05$ & $1.10959 \mathrm{E}-04$ & $3.30406 \mathrm{E}-05$ \\
\hline .8 & $2.56000 \mathrm{E}-04$ & $2.73278 \mathrm{E}-04$ & $1.72775 \mathrm{E}-05$ & $2.24550 \mathrm{E}-04$ & $3.14502 \mathrm{E}-05$ \\
\hline 1 & $4.00000 \mathrm{E}-04$ & $3.63253 \mathrm{E}-04$ & $3.67468 \mathrm{E}-05$ & $3.08591 \mathrm{E}-04$ & $9.14093 \mathrm{E}-05$ \\
\hline
\end{tabular}

(Table (1) describes the exact and numerical solution of Eq. (4.1) when $k(|x-y|)=|x-y|^{-v} ; 0<v<1$, at $\lambda=0.5152 ; \mu=1 ; T=0.2 ; n=41 ;$ for $v=0.12$ and $v=0.73$ ) 
We can deduce from the above and other results the following:

1) When the values of $\lambda$ and $T$ are fixed, the error increases with increasing of $v$, where $v$ is called Poisson ratio, in the theory of elasticity and when $0<v<0.5$, the atomic bond between the particles of the material is normal, while $v \geq 0.5$ the atomic bond is strong, for this the error may be large .

2) It was found that the highest error value is obtained when $v=0.73$ at $x= \pm 1$. Also, the errordecreases gradually, and has less valuewhen $=0.07$.

3) When the values of $\lambda$ and $v$ are fixed,the error value increases with the time.

4) The maximum error is 0.2363917451 , at $x= \pm 1$, when $v=0.73, T=0.8$.

5) The minimum error is $1.81871 \mathrm{E}-7$, at $x= \pm 0.0$, when $v=0.07, T=0.004$.

6) In all the studied situations, the error value increases when it get closer to the ends points $x= \pm 1$. It also decreases at the middle when it gets closer to zero.

Example (2): Consider the logarithmic form $k(|x-y|)=\ln |x-y|$, at $\mu=1, \lambda=.25 n=41, \quad T=0.2$.

Table 2

\begin{tabular}{|l|l|l|l|}
\hline$x$ & Exact & App. & Error \\
\hline-1 & $4.00 \mathrm{E}-04$ & $4.14364 \mathrm{E}-04$ & $1.43636 \mathrm{E}-05$ \\
\hline-0.8 & $2.56 \mathrm{E}-04$ & $2.53924 \mathrm{E}-04$ & $2.07618 \mathrm{E}-06$ \\
\hline-0.6 & $1.44 \mathrm{E}-04$ & $1.43201 \mathrm{E}-04$ & $7.98768 \mathrm{E}-07$ \\
\hline-0.4 & $6.40 \mathrm{E}-05$ & $6.37610 \mathrm{E}-05$ & $2.38987 \mathrm{E}-07$ \\
\hline-0.2 & $1.60 \mathrm{E}-05$ & $1.60212 \mathrm{E}-05$ & $2.12470 \mathrm{E}-08$ \\
\hline 0 & $0.00 \mathrm{E}+00$ & $9.86122 \mathrm{E}-08$ & $9.86122 \mathrm{E}-08$ \\
\hline 0.2 & $1.60 \mathrm{E}-05$ & $1.60212 \mathrm{E}-05$ & $2.12433 \mathrm{E}-08$ \\
\hline 0.4 & $6.40 \mathrm{E}-05$ & $6.37610 \mathrm{E}-05$ & $2.38987 \mathrm{E}-07$ \\
\hline 0.6 & $1.44 \mathrm{E}-04$ & $1.43201 \mathrm{E}-04$ & $7.98770 \mathrm{E}-07$ \\
\hline 0.8 & $2.56 \mathrm{E}-04$ & $2.53924 \mathrm{E}-04$ & $2.07618 \mathrm{E}-06$ \\
\hline 1 & $4.00 \mathrm{E}-04$ & $4.14364 \mathrm{E}-04$ & $1.43636 \mathrm{E}-05$ \\
\hline
\end{tabular}

(Table (2) describes the exact and numerical solution of Eq. (4.1)

when $k(|x-y|)=\ln |x-y|$, at $\lambda=0.25 ; \mu=1 ; T=0.2 ; n=41$.)

We notice from the results of the program at the previous and others cases that

1) When the values of $T$ are fixed,the error values clearly increase with increasing of $\lambda$.

2) When the values of $\lambda$ are fixed,the error values increase with the time increase.

3) The maximum error is 0.020521631 , at $x= \pm 0.8$, when $\lambda=0.4368, T=0.8$.

4) The minimum error is $8.4973 \mathrm{E}-10$, at $= \pm 0.2$, when $\lambda=0.25, T=0.004$.

5) The error value increases when it get closer to the ends points $x= \pm 1$. It decreases at the middle when it gets closer to zero.

\section{References}

[1]. T. Allahviranloo, Z. Gouyandeh, A. Armand, Numerical solutions for fractional differential equations by Tau-Collocation method, Appl. Math.and Compute.271(2015)979-990.

[2]. F. Ghoreishi, M. Hadizadeh, Numerical computation of the Tau approximation for the Volterra-Hammerstein integral equations, Numer.Algor.(2009) 52:541559.

[3]. H.R. Marzban, H.R. Tabrizidooz, M. Razzaghi, A composite collocation method for the nonlinear mixed Volterra-FredholmHammerstein integral equations, Commun. Non. Sci. Numer. Simul.16 (2011) 1186-1194.

[4]. ] H.L. Dastjerdia, F.M.M. Ghainia, M. Hadizadeh, A meshless approximate solution of mixedVolterra-Fredholm integral equations, Inter. J. Comput. Math., 90 (2013) 527-538.

[5]. C.D.Green,Integral Equation Methods,Nelson, New York, 1969

[6]. H. Hochstadt,Integral Equations, A Wiley Inter Science Publication, New York, 1973.

[7]. R. P.Kanwal,Linear Integral Equations Theory and Technique,Boston, 1996

[8]. S. Schiavone, A.R.Constanta and L.Y. Mioduchowski,Integral Methods in Science and

[9]. Engineering, BirkhauserBoston, 2002.

[10]. Jawsan and Symm,Integral Equation Method in Potential Theory andElastostatics, Academic press,London, 1977.

[11]. David Colton and RainerKress,Integral Equation Methods in Scattering Theory, A Wiley.Interscience Publication John Wiley, 1983

[12]. W. E. Olmstoad and A. K.Goutesen. Integral representation and Oseen flow problems, Mech.Today, (1976) 125 - 185 M. A. Abdou, A. A. Badr, On a method for solving an integral equation in the displacement contact problem. Appl. Math. Compute. 127 (2002) $65-72$

[13]. M. A. Abdou, O. L. Mustafa, Fredholm - Volterra integral equation in contact problem, Appl. Math. Compute. 138 (2003), 199 215

[14]. M. A. Abdou, F. A. Salama, Volterra - Fredholm integral equation of the first kind and spectral relationships, Appl. Math. Compute. 153 (2004), 141 - 153.

[15]. M. A.Abdou,Fredholm - Volterra integral equation and generalized potential kernel. Appl. Math. Compute. 131 (2002) 81 - 94 
[16]. M. A. Abdou, M. S.Ismail,Toeplitz matrix and product Nystrom methods for solving the singular integral equation, Le Matematica Vol. LVII ( 2002 ) - FascLPP. 21 - 37

[17]. M. A. Abdou, Mohamed and Ismail,On the numerical solutions of integral equation of mixed type, Appl. Math. Compute. 138, (2003) $172-186$

[18]. K.E. Atkinson,The Numerical Solution of Integral Equation of the Second Kind, Cambridge University,Cambridge, 1997

[19]. M. A. Abdou, M. M. EL-Borai, M. M. El-Kojok, Toeplitz matrix method and nonlinear integral equation of Hammerstein type, J. Comp. Appl. Math.Vol. 223, (2009) $765-776$ 